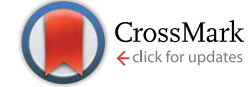

Cite this: Analyst, 2014, 139, 5350

Received 29th July 2014

Accepted 25th August 2014

DOI: 10.1039/c4an01393j

www.rsc.org/analyst

\section{Acoustic monitoring of changes in well-defined hyaluronan layers exposed to chondrocytes $\dagger$}

\author{
E. Nilebäck, ${ }^{a b}$ L. Enochson, ${ }^{c}$ N. Altgärde, ${ }^{a}$ M. Schnabelrauch, ${ }^{d}$ A. Lindahl, ${ }^{c}$ \\ S. Svedhem ${ }^{\star a}$ and A. Kunze ${ }^{\star a e}$
}

The interaction of human-derived chondrocytes and thin hyaluronan layers was studied using the quartz crystal microbalance with dissipation (QCM-D) technique combined with light microscopy. This approach allowed unique real-time monitoring of the interface between the cells and the sensor surface. Our results suggest that the hyaluronan layer is rapidly degraded by chondrocytes.

Biocompatibility is critical in developing new materials for implants. Most commonly, in vitro animal models or in vivo human models are required for biocompatibility testing of new materials, e.g. checking for signs of inflammation or foreign body reactions. ${ }^{1}$ The observed responses from these animal or human models are closely related to processes taking place at the cell-material interface. Thus, surface-based in vitro evaluation techniques are advancing in this field of research. In particular, the study of material-cell interactions using surfacebased analytical techniques allows for a more detailed understanding of the properties of the interface between the tissue and the biomaterial, down to the nm-level. In this study, an approach to study the nano-mechanical properties of the interface between human-derived chondrocytes and hyaluronan (HA) based on the quartz crystal microbalance with dissipation (QCM-D) technique combined with light microscopy is demonstrated (see schematic illustration in Fig. 1).

HA is a glycosaminoglycan (GAG) built up as a biopolymer by repeating disaccharide units with varying molecular weights up to several MDa. Together with the proteoglycan aggrecan, HA is

${ }^{a}$ Dept. of Appl. Physics, Chalmers University of Technology, 412 96, Göteborg, Sweden. E-mail: sofia.svedhem@chalmers.se

${ }^{b}$ Biolin Scientific AB, 426 77, Västra Frölunda, Sweden

${ }^{c}$ Dept. of Clinical Chemistry and Transfusion Medicine, Sahlgrenska Academy, University of Gothenburg, 413 45, Göteborg, Sweden

${ }^{d}$ Biomaterials Department, INNOVENT e.V., 07745 Jena, Germany

${ }^{e}$ Inst. of Physical Chemistry, Univerisity of Götttingen, 37077, Göttingen, Germany. E-mail: angelika.kunze@chemie.uni-goettingen.de

$\dagger$ Electronic supplementary information (ESI) available. See DOI: 10.1039/c4an01393j one of the main components in the extra cellular matrix (ECM) of mammalian cells where it is active in both signalling and the structural integrity of the ECM. ${ }^{2}$ In the ECM, HA is constantly remodelled by surrounding cells, e.g., chondrocytes, that express enzymes such as hyaluronidase. ${ }^{3}$ Particular abundance of HA is found in articular cartilage tissue where it acts as a coat surrounding chondrocytes being one of the main components responsible for the high compression resistance of cartilage. Being a biological, abundant and non-toxic material, HA has been introduced as biomaterial acting as a scaffold for tissue regeneration of cartilage and skin. ${ }^{4,5}$ However, the nature of the underlying interaction between the cells and the HA is still not (fully) understood because most commonly used approaches such as optical microscopy do not allow for the study of processes directly at the interface of the cells and the interacting biomaterial. An attractive approach to study this interaction is employing surface-based analytical techniques. These require immobilization of HA onto the sensor surface without the loss of bioactivity of the GAG. In a recent study, we describe grafting of biotinylated HA on gold-coated sensor surfaces. Retained bioactivity of end-on immobilized HA was verified by monitoring the enzymatic degradation of HA by hyaluronidase and the HA interaction with aggrecan. ${ }^{6}$ This bottom-up approach has resulted in a detailed understanding for the preparation of

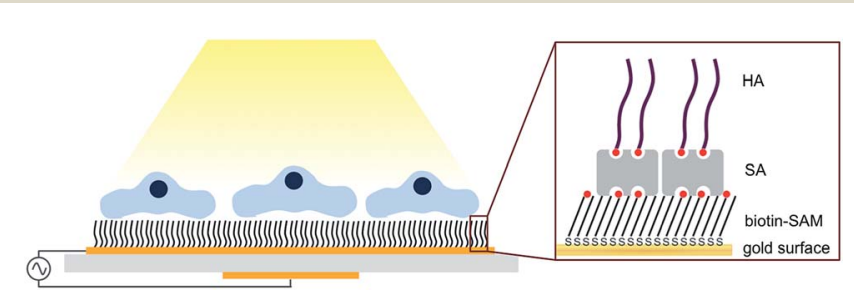

Fig. 1 Schematic illustration of the experimental design, using a combined set-up with a windowed QCM-D module mounted on a light microscope, and the immobilization of end-on biotinylated HA. $\mathrm{HA}$ is immobilized onto a streptavidin (SA) layer bound to a biotinylated thiol self-assembly monolayer (biotin-SAM) on a gold-coated sensor surface. Image is not drawn to scale. 
well-defined layers of HA while minimizing unspecific binding and, most importantly, preserving the bioactivity of the GAG. Here, the same surface chemistry is applied for the study of the interaction of HA with chondrocytes using the QCM-D technique in combination with light microscopy.

QCM-D is an acoustic technique that measures changes in mass and viscoelastic properties of a material close $(<250 \mathrm{~nm}$ in water and no-slip conditions) to the sensor surface. ${ }^{7}$ While traditionally more focused on protein adsorption and interaction studies in the field of biomaterials, QCM-D is increasingly being used to study cell-surface interactions and cell morphology. ${ }^{8-10}$ The implementation of QCM-D in cell studies reveals besides the information about the cell-surface interaction also information about the mechanical properties of the cells at the focal adhesion points. ${ }^{\mathbf{1 1}}$ Furthermore it has been suggested, that the strength of the cell-surface bond, i.e., the mechanical and acoustical coupling between the cell and the oscillating sensor, determines the QCM-D response. ${ }^{12}$ Those studies have demonstrated that QCM-D is very well suited for the study of biomolecular interfacial layers and their effect on cell adhesion.

\section{Results and discussion}

With our study we aimed to elucidate the processes occurring at the interface between human-derived chondrocytes and surface-immobilized HA layers. For this purpose a QCM-D instrument (Q-Sense E1, Biolin Scientific, Västra Frölunda, Sweden) equipped with a window module that allows for simultaneous light microscopy imaging of the cells was employed. In a typical QCM-D experiment, chondrocytes were injected at a high concentration of 750000 cells $/ \mathrm{mL}$ in serum free media and at a high flow rate $(300 \mu \mathrm{L} / \mathrm{min})$. The influx of the cells into the QCM-D chamber was observed with the light microscope equipped with a $10 \times$ objective. When the chamber was completely filled with cell suspension the flow was stopped to allow the chondrocytes to attach and spread onto the sensor surface (experimental details see ESI $\dagger$ ).

In general, when studying cell attachment by QCM-D, one would possibly expect a negative frequency shift due to mass load by the cells, and a positive dissipation shift due to the energy that is dissipated by attached cells during the oscillation of the quartz crystal. Such behaviour was observed when chondrocytes were added in serum-free cell culture media to sensors coated with polystyrene, which is a known cell adhesive material. Fig. 2 shows the QCM-D frequency and dissipation curves versus time as well as live light microscopy images taken during the attachment of chondrocytes to a polystyrene coated sensor surface. The addition of chondrocytes to the sensors resulted in negative frequency shifts of $\Delta f=-(37 \pm 10) \mathrm{Hz}$ and positive dissipation shifts of $\Delta D=(13 \pm 16) \times 10^{-6}$ (statistics see ESI $\dagger$ ). The large variance in these dissipation shifts is most likely attributed to a difference in cell coverage and the presence of aggregated cells on the different sensors. After approximately one hour, cell spreading started as observed with the light microscope (see microscopy image taken at $t=65 \mathrm{~min}$ in Fig. 2). At the same time, the dissipation shift started to decrease whilst

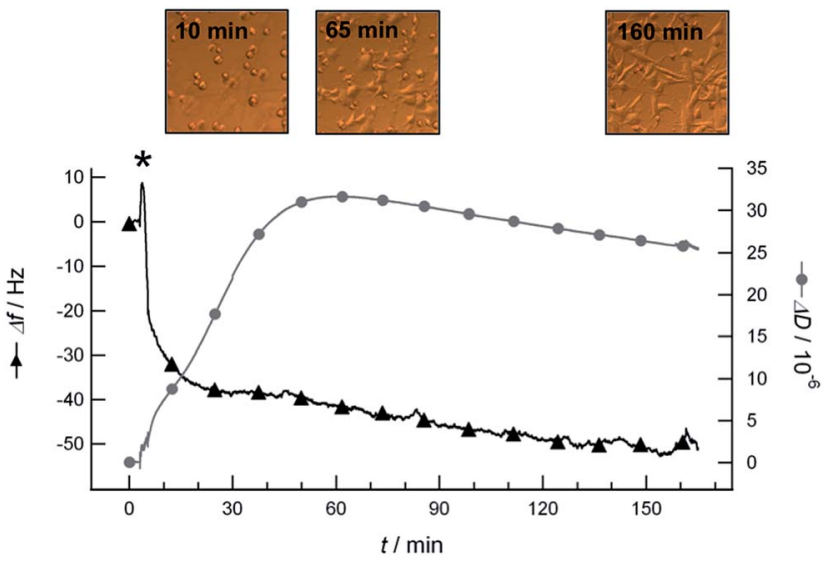

Fig. 2 Frequency ( $\Delta f$, black triangles) and dissipation ( $\Delta D$, grey circles) shifts for the addition of chondrocytes to a polystyrene coated sensor and corresponding light microscopy image taken at $t=10 \mathrm{~min}, t=65$ $\mathrm{min}$, and after rinsing at $t=160 \mathrm{~min}$. The asterisk indicates the addition of cells at $t=6 \mathrm{~min}$.

the frequency shift continued decreasing. This decrease of the dissipation shift can be explained by the shape transformation that the cells undergo during spreading..$^{13}$ After $\approx 160 \mathrm{~min}$, we observed all cells to be spread over the entire sensor surface and to remain attached after rinsing with medium.

In contrast to the results obtained for the attachment of chondrocytes on polystyrene coated sensors are the observations made when the cells are injected to sensors coated with HA. In this case the frequency shift was observed to increase whilst the dissipation shift decreased after injection of cells to the HA coated QCM-D sensor (Fig. 3).

In Fig. 3 the immobilization steps of end-on biotinylated HA on the sensor surface and the subsequent addition of chondrocytes in serum-free cell culture medium are shown. In the first step (Fig. 3, step i), streptavidin is bound to the sensor surface that had been functionalized ex situ with a biotinylated thiol SAM (composed of oligo-ethylene glycol disulfides, with terminal -OH (99\%) and biotin (1\%) groups) prior to the QCMD experiment (for details see ESI $\dagger$ ). ${ }^{\mathbf{1 4}}$ A typical frequency shift about $23 \mathrm{~Hz}$ together with essentially no shift in dissipation is observed upon binding of streptavidin. ${ }^{14}$ In the following step (Fig. 3, step ii), end-on biotinylated HA (MW $23 \mathrm{kDa}$ ) is added resulting in a frequency shift about $24 \mathrm{~Hz}$ and a dissipation shift of about $6.5 \times 10^{-6}$ as reported previously. ${ }^{6}$ After successful immobilization of HA on the QCM-D sensor, chondrocytes are injected (Fig. 3, indicated by the asterisk) using the same experimental conditions as above (for the attachment on polystyrene coated sensors). As shown in Fig. 3, a decrease in dissipation and an increase in the frequency shift were observed upon addition of chondrocytes (Fig. 3), which is opposite to the behaviour presented in Fig. 2 for the attachment of chondrocytes on polystyrene coated sensors. The QCM-D signal stabilized at $\approx 100 \mathrm{~min}$ after injection of chondrocytes. The final decrease in frequency shift and increase in dissipation shift during this time span corresponds to $89.6 \pm 3.9 \%$ of the value obtained for the immobilization of HA (indicated by the dotted line in Fig. 3). 


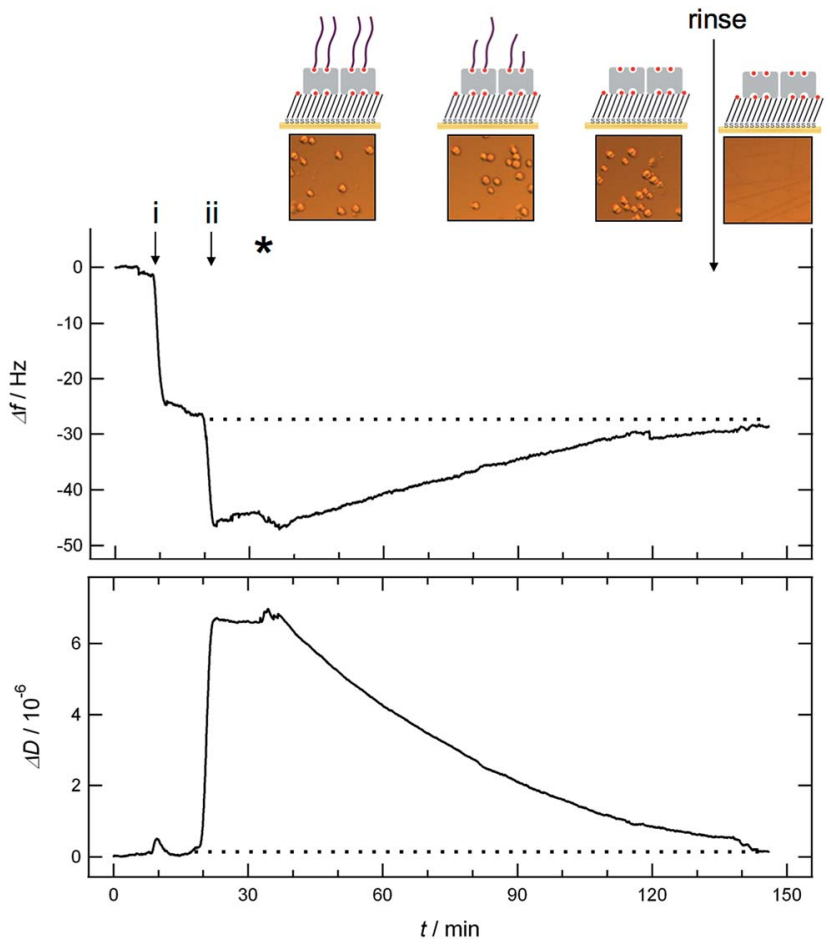

Fig. 3 QCM-D frequency ( $\Delta f$, middle row) and dissipation $(\Delta D$, bottom row) shifts for the addition chondrocytes (marked by an asterisk) to immobilized HA. (i) Addition of SA followed by (ii) addition of HA. Top row: schematic illustration of the suggested degradation process and light microscopy images taken during the QCM-D experiment during incubation with cells and after rinsing with media.

Because neither attachment nor spreading of the cells onto the HA coated surface could be observed with light microscopy and as all cells were removed upon rinsing in the end of the experiment $(t=140 \mathrm{~min})$, we assume the observed shifts in frequency and dissipation to be due to degradation of the immobilized HA by the chondrocytes. Under this assumption the observed shifts correspond to a degradation of the HA by $\approx 90 \%$ (see Fig. 4).

Comparing our findings with earlier results obtained for the degradation of HA layers by hyaluronidase, ${ }^{6}$ we note that the

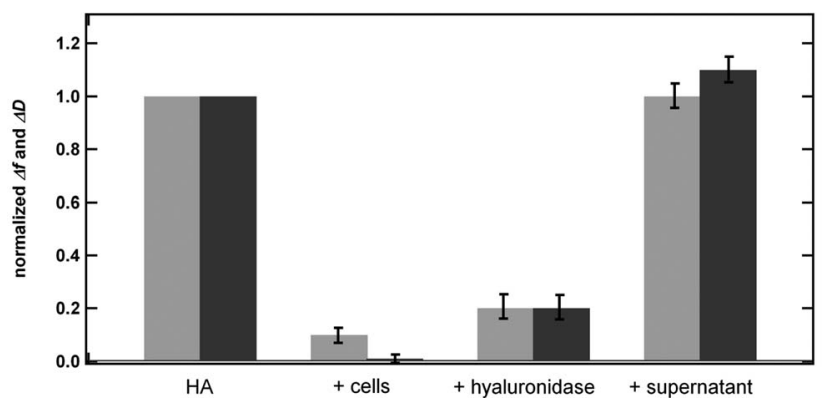

Fig. 4 Normalized QCM-D frequency (light grey) and dissipation shift (dark grey) for a HA layer ( $\Delta f$ and $\Delta D$ for the HA layer is set to 1 ) and after the subsequent addition of either cells, hyaluronidase (data taken from ref. 6) or supernatant. frequency and dissipation shifts are essentially the same for the two cases (see Fig. 4). However, in comparison to the degradation process by hyaluronidase, HA is degraded with slower kinetics by the chondrocytes, most likely due to a lower concentration of enzymes secreted by or present in the glycocalix of the cells compared to the experiments with the purified enzyme.

Control experiments were performed to verify that the observed QCM-D signal is due to an interaction between the cells and the HA layer and not due to (i) the action of enzymes dissolved in the supernatant of the chondrocytes, or (ii) an interaction between the cells and the SA layer underneath the HA.

Degradation of HA by enzymes in the supernatant can be excluded because no change in the QCM-D signal was observed upon rinsing the HA layer with the supernatant of the chondrocytes for $2 \mathrm{~h}$ (see Fig. 4 and ESI $\dagger$ ). Similarly, an interaction between the chondrocytes and SA can be excluded, since no change in the QCM-D signal was observed upon addition of cells to SA-coated QCM-D sensors. The presence of chondrocytes on top of the SA layer was verified by light microscopy, but neither attachment nor spreading of the cells was observed. Upon rinsing the sensor with media, all chondrocytes were removed from the QCM-D chamber (see ESI $\dagger$ ). These experiments support our assumption that the observed QCM-D results in Fig. 3 are due to a degradation process of HA caused by the chondrocytes in solution.

Another possible explanation for the observed QCM-D signal during exposure of the HA layer to chondrocytes could be a deswelling of the highly hydrated biopolymer. ${ }^{6}$ However, deswelling or collapsing is usually induced by drastic changes in $\mathrm{pH}$, ionic strength or temperature, which are all parameters that are under given experimental conditions essentially constant. Thus, we largely exclude a deswelling of the HA layer to be the underlying process for the observed QCM-D signal.

We would also like to briefly discuss how the cells are (acoustically) coupled to the QCM-D sensor for the two different surfaces, the polystyrene and the HA coated sensors. In the case of polystyrene, the attachment of chondrocytes in serum-free medium induced a negative frequency shift and positive dissipation shift, which correspond to a tightly bound, soft layer. This indicates that cells come in close proximity to the sensor surface and couple directly to the oscillatory motion of the quartz crystal inducing an increase in mass and energy dissipation. Images taken with light microscopy show cells to attach and spread onto the sensor surface, which supports this assumption. We want to note that the experiments were performed in serum-free medium. If serum-containing medium would be used, serum proteins would adsorb prior to the cells onto the surface, building a soft protein cushion that most likely would affect the acoustic coupling and thus the QCM-D signal. ${ }^{12}$

In the case of the HA-coated sensor surface chondrocytes are not directly sensed by QCM-D despite their presence in solution above the sensor surface (as verified by light microscopy). This means that cells are not acoustically coupled to the QCM-D sensor because they are either simply outside the penetration 
depth of the shear wave (sensing depth, approx. $250 \mathrm{~nm}$ in water) or because the chondrocytes are bound very weakly to the sensor similar to our recent observations made for the attachment of platelets to plasma proteins. ${ }^{12}$ In fact, that cells are not acoustically sensed provides the benefit that the observed signal is directly related to processes occurring at the interface between the cells and the underlying biomaterial.

\section{Conclusions}

Using acoustic sensing combined with a defined immobilization of HA to the sensor surface, we observed for the interaction between HA layers and chondrocytes, a signal corresponding to the removal of the HA layer, suggesting an active degradation caused by enzymes secreted by or present in the glycocalix of the chondrocytes. These results show that acoustical sensing is a powerful method to monitor in real-time and label-free degradation or other biochemical processes taking place at the interface between cells and a biomaterial surface.

\section{Acknowledgements}

Financial support was received from the European Union Seventh Framework Program (FP7/2007-2013) under grant agreement no NMP4-SL2009-229292 ("Find \& Bind").

\section{Notes and references}

1 J. M. Anderson, Annu. Rev. Mater. Res., 2001, 31, 81-110.
2 M. A. Solis, Y. H. Chen, T. Y. Wong, V. Z. Bittencourt, Y. C. Lin and L. L. H. Huang, Biochem. Res. Int., 2012, DOI: $10.1155 / 2012 / 346972$.

3 E. R. Bastow, S. Byers, S. B. Golub, C. E. Clarkin, A. A. Pitsillides and A. J. Fosang, Cell. Mol. Life Sci., 2008, 65, 395-413.

4 G. Kogan, L. Soltes, R. Stern and P. Gemeiner, Biotechnol. Lett., 2007, 29, 17-25.

5 T. Dehne, C. Karlsson, J. Ringe, M. Sittinger and A. Lindahl, Arthritis Res. Ther., 2009, 11, DOI: 10.1186/ar2800.

6 N. Altgärde, E. Nilebäck, L. De Battice, I. Pashkuleva, R. L. Reis, J. Becher, S. Möller, M. Schnabelrauch and S. Svedhem, Acta Biomater., 2013, 9, 8158-8166.

7 M. Rodahl, F. Hook, A. Krozer, P. Brzezinski and B. Kasemo, Rev. Sci. Instrum., 1995, 66, 3924-3930.

8 E. Nileback, F. Westberg, J. Deinum and S. Svedhem, Anal. Chem., 2010, 82, 8374-8376.

9 J. Y. Chen, A. Shahid, M. P. Garcia, L. S. Penn and J. Xi, Biosens. Bioelectron., 2012, 38, 375-381.

10 E. Watarai, R. Matsuno, T. Konno, K. Ishihara and M. Takai, Sens. Actuators, B, 2012, 171-172, 1297-1302.

11 M. P. Garcia, A. Shahid, J. Y. Chen and J. Xi, Anal. Bioanal. Chem., 2012, 1-6.

12 A. Kunze, C. Hesse and S. Svedhem, Colloids Surf., B, 2014, 116, 446-451.

13 N. Tymchenko, E. Nilebäck, M. Voinova, J. Gold, B. Kasemo and S. Svedhem, Biointerphases, 2012, 7, 1-9.

14 E. Nilebäck, L. Feuz, H. Uddenberg, R. Valiokas and S. Svedhem, Biosens. Bioelectron., 2011, 28, 407-413. 